\title{
Efeitos da adoção do modelo do Prêmio Nacional da Qualidade na medição de desempenho: estudos de caso em empresas ganhadoras do prêmio
}

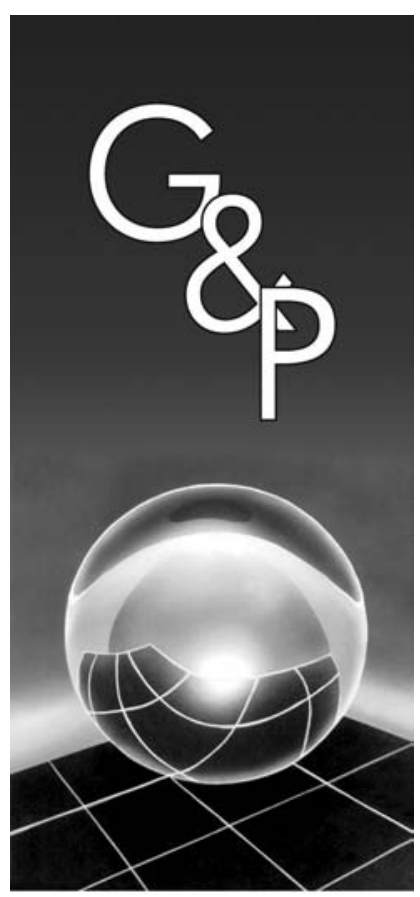

Gerusa Tinasi de Oliveira

Roberto Antonio Martins

\section{Resumo}

Este artigo tem por objetivo apresentar os resultados de um estudo de caso múltiplo sobre os efeitos da adoção do Prêmio Nacional da Qualidade (PNQ) na medição de desempenho. A pesquisa empírica foi realizada em três das quatro empresas ganhadoras do PNQ no ano de 2005. Os principais resultados apontam que o modelo de excelência do prêmio exerce um papel importante tanto com uma alavanca externa como interna na evolução da medição de desempenho das empresas estudadas. Os critérios de excelência "estratégia e planos" e "informação e conhecimento" agem como alavancas externas. Já a implementação ou melhoria de práticas de gestão atuam como alavancas internas para modificação da medição de desempenho nos estágios de reflexão, modificação e desdobramento. As mudanças nos sistemas de desempenho ocorreram em graus diferentes nas empresas estudadas.

Palavras-chave: Medição de desempenho. Prêmio Nacional da Qualidade. Sistemas de medição de desempenho. Modelos de excelência na gestão.

\section{Introdução}

O ambiente em que a maioria das organizações compete é dinâmico e as rápidas mudanças, que nele ocorrem, requerem constantemente estratégias e operações que possam refletir essas circunstâncias ambientais (KENNERLEY et al., 2003). Pautadas por essas novas estratégias, muitas empresas vêm investindo recursos em tecnologia e novas práticas de gestão para sobreviverem no mercado atual ou conquistarem novos mercados.

Diante da necessidade de melhorar a qualidade dos produtos e, conseqüentemente, aumentar a competitividade das empresas, em meados da década de 80 um grupo de especialistas norte-americanos analisou diversas organizações consideradas de sucesso com o objetivo de encontrar nelas características comuns que as diferenciassem das demais. Assim, surgiram os prêmios de excelência em qualidade e negócios que reconhecem o desempenho organizacional, um componente significativo das estratégias de produtividade e promoção da qualidade em muitos países (MIGUEL, 2004). No Brasil, o Prêmio Nacional da Qualidade (PNQ) é administrado pela Fundação Nacional da Qualidade (FNQ) desde
1991, sendo um prêmio de reconhecimento à excelência na gestão das organizações.

A medição de desempenho pode ser modificada por forças externas, como por exemplo, a competitividade do mercado e a adoção de um novo modelo de gestão como, por exemplo, o modelo de excelência do PNQ. Como tal modelo possui critérios específicos sobre a medição de desempenho, sua adoção pode requerer novas práticas de medição de desempenho, desencadeando um processo de evolução por meio da reflexão, modificação e desdobramento de um novo sistema de medição de desempenho (SMD).

Neste sentido, o objetivo deste artigo é investigar empiricamente como as empresas ganhadoras do PNQ modificaram suas práticas de medição de desempenho após adoção do modelo de excelência do prêmio. $\mathrm{O}$ presente artigo está estruturado da seguinte forma: inicialmente é realizada uma revisão bibliográfica sobre medição de desempenho e PNQ. Em seguida, o método de pesquisa e os resultados da pesquisa de campo são apresentados. Finalizando, algumas considerações são 
feitas sobre os resultados encontrados à luz da teoria estudada.

\section{Medição de desempenho}

Antes de apresentar o conceito de medição de desempenho é importante tratar do conceito de desempenho. De maneira geral, pode ser percebido que as definições de desempenho encontradas na bibliografia pesquisada parecem se justapor e convergir para a de Lebas (1995, p. 29). Esse autor afirma que o desempenho é sumarizado como:

\section{“... gestão e desdobramento dos componentes do modelo causal que levam ao alcance dos objetivos declarados, dentro das restrições espe- cíficas (...) para os tomadores de decisão...” (LEBAS, 1995, p. 29).}

Desta forma, a definição de desempenho de Lebas (1995) pode ser utilizada para compreender melhor a medição de desempenho.

Neely et al. (1995) descrevem a medição de desempenho como um processo de quantificar a ação. Neely (1998, p.5) define

“... um sistema de medição de desempenho que possibilita que decisões e ações sejam tomadas com base em informações porque ele quantifica a eficiência e a eficácia de decisões passadas por meio da aquisição, compilação, arranjo, análise, interpretação e disseminação de dados adequados." (NEELY, 1998, p. 5)

Com uma visão mais técnica, Kueng et al. (2000) afirmam que o sistema de medição de desempenho (SMD) pode ser entendido como um sistema de tecnologia de informação que coleta dados relevantes de desempenho a partir de várias fontes, compara dados atuais com dados históricos e metas, e comunica os resultados para os usuários.

Para Kennerley et al. (2003), o SMD é o inter-relacionamento de três elementos: medidas singulares que quantificam o impacto de ações específicas, um conjunto de medidas que são combinadas para avaliar o desempenho da organização como um todo, e uma infra-estrutura de suporte que permite que dados sejam obtidos, coletados, classificados, analisados, interpretados e disseminados para o uso gerencial.

Franco-Santos et al. (2004), a partir de uma revisão bibliográfica extensa, definem medição de desempenho como

“...o conjunto de processos que uma organização usa para gerenciar a implementação da sua estratégia, comunicar sua posição e progresso, e influenciar o comportamento e ações dos seus funcionários. Isso requer a identificação dos objetivos estratégicos, medidas de desempenho multidimensionais, metas e o desenvolvimento de uma infra-estrutura de suporte." (FRANCOSANTOS et al., 2004)

As medidas de desempenho, segundo Hronec (1994), são como "sinais vitais" da organização, pois quantificam as atividades críticas dentro de processos-chave de produção; proporcionam a comunicação da estratégia da empresa para os níveis inferiores da hierarquia, propiciando à organização o conhecimento de quais processos-chave devem apresentar resultados mais satisfatórios para que a empresa alcance suas metas; e interligam a missão, a estratégia, as metas aos processoschave e às atividades críticas da empresa.

$\mathrm{Na}$ bibliografia pesquisada, diversos autores propõem classificações para as medidas de desempenho. White (1996) as classifica nas seguintes categorias: fonte dos dados (interna ou externa); tipo de dado (subjetivo ou objetivo); referência (benchmark ou self-referenced); e orientação do processo (entrada ou saída). Entretanto, existem outras classificações como: financeiros e não-financeiros (KAPLAN; NORTON, 1992; KAYDOS, 1991; KEEGAN et al., 1989; McNAIR et al., 1990); global e local (MASKELL, 1991); e satisfação dos stakeholders da empresa (ATKINSON et al., 1997; MARTINS; COSTA NETO, 1998).

De forma a agrupar os novos SMD's, Wongrassamee et al. (2003) sugerem duas categorias distintas. A primeira é referente aos sistemas de medição de desempenho que enfatizam a auto-avaliação como, por exemplo, o Deming Prize (Japão e Ásia); o Malcolm Baldrige National Quality Award (EUA) e o The European Foundation for Quality Management Award (Europa). A segunda categoria é aquela dos SMD's projetados para ajudar os gerentes a medirem e melhorarem os processos de negócio. São eles: CapabilityMaturityMatrices (Software Engineering Institute); The Performance Pyramid; The Effective Progress and Performance Measurement (EP2M) e o Balanced Scorecard. Além desses, outros exemplos de SMD's para medição e melhoria de processos podem ser citados, tais como: SMART/Performance Pyramid (CROSS; LYNCH, 1990); Integrated Performance Measurement System (BITITCI et al., 1997); Medição de desempenho para World Class Manufacturing (MASKELL, 1991); Cambridge Performance Measurement Design Process (NEELY et al., 1995) e Performance Prism (NEELY; ADAMS, 2001). Vale destacar que o Balanced Scorecard, proposto por Kaplan e Norton $(1992,1997)$, é o modelo recomendado pelo Comitê de Planejamento do sistema de medição do desempenho global constituído pela Fundação Nacional para Qualidade (FPNQ, 2001) para o desenvolvimento e implementação do modelo de medição de desempenho do Prêmio Nacional da Qualidade, tema de pesquisa deste artigo. 
Vários autores identificam os propósitos e as justificativas para o uso dos novos sistemas de medição de desempenho. Para Martins (1998), o uso de SMD's é categorizado do seguinte modo: maneiras diferentes para compartilhar a informação para controlar e melhorar o desempenho; indução das atitudes dos empregados; estabelecimento do relacionamento de causa e efeito entre as medidas de desempenho; e maneiras diferentes de analisar a informação.

Neely (1998) propõe que a utilização de um SMD por quatro motivos, os denominados 4 CP's: conferir a posição pelo estabelecimento de posição no mercado, o uso do benchmarking para comparar posições com competidores e monitorar o progresso; comunicar a posição para divulgar o desempenho para toda a organização e os reguladores (governo, acionistas e sociedade em geral); confirmar as prioridades para a ação e verificação da alta administração, para clarificar a tomada de decisão e como significado para gestão, controle de custos e investimento; e compelir o progresso como significado para motivação, comunicação das prioridades da organização e como base para recompensa.

Assim, o papel do uso da informação sobre desempenho é relevante para guiar o processo de concepção de sistemas de medição de desempenho. Esse mesmo autor afirma que as necessidades de informação sobre desempenho dos vários usuários do sistema são os principais direcionadores para o desenvolvimento de um SMD (MARTINS, 2002).

Neste contexto, é de grande importância a evolução dos sistemas de medição de desempenho para garantir que eles continuem a refletir o ambiente e os objetivos da organização (KENNERLEY; NEELY, 2002). Do mesmo modo, Waggoner et al. (1999) afirmam que a mudança e a evolução dos SMD's podem ser impulsionadas por quatro categorias de forças, como: influências internas (relações de poder e interesses da coalizão dominante); influências externas (legislação e volatilidade do mercado); assuntos relacionados aos processos (maneiras de implementação e gestão de processos políticos); e assuntos relacionados à transformação organizacional (o grau de suporte da alta administração e risco de ganho ou perda da mudança).

Kennerly e Neely (2002) destacam que para gerir efetivamente a evolução de um sistema de medição de desempenho, a organização deve levar em conta algumas considerações como: o uso de sistemas de medição de desempenho como um pré-requisito para a evolução; o SMD como o inter-relacionamento de três elementos proposto por Neely et al. (1995) e a existência de alavancas internas e externas.

Além disso, esses autores propõem um modelo do processo de evolução para gerenciar os SMD's de forma que eles se mantenham fornecendo dados e informações relevantes para a tomada de decisão, como ilustrado na Figura 1.

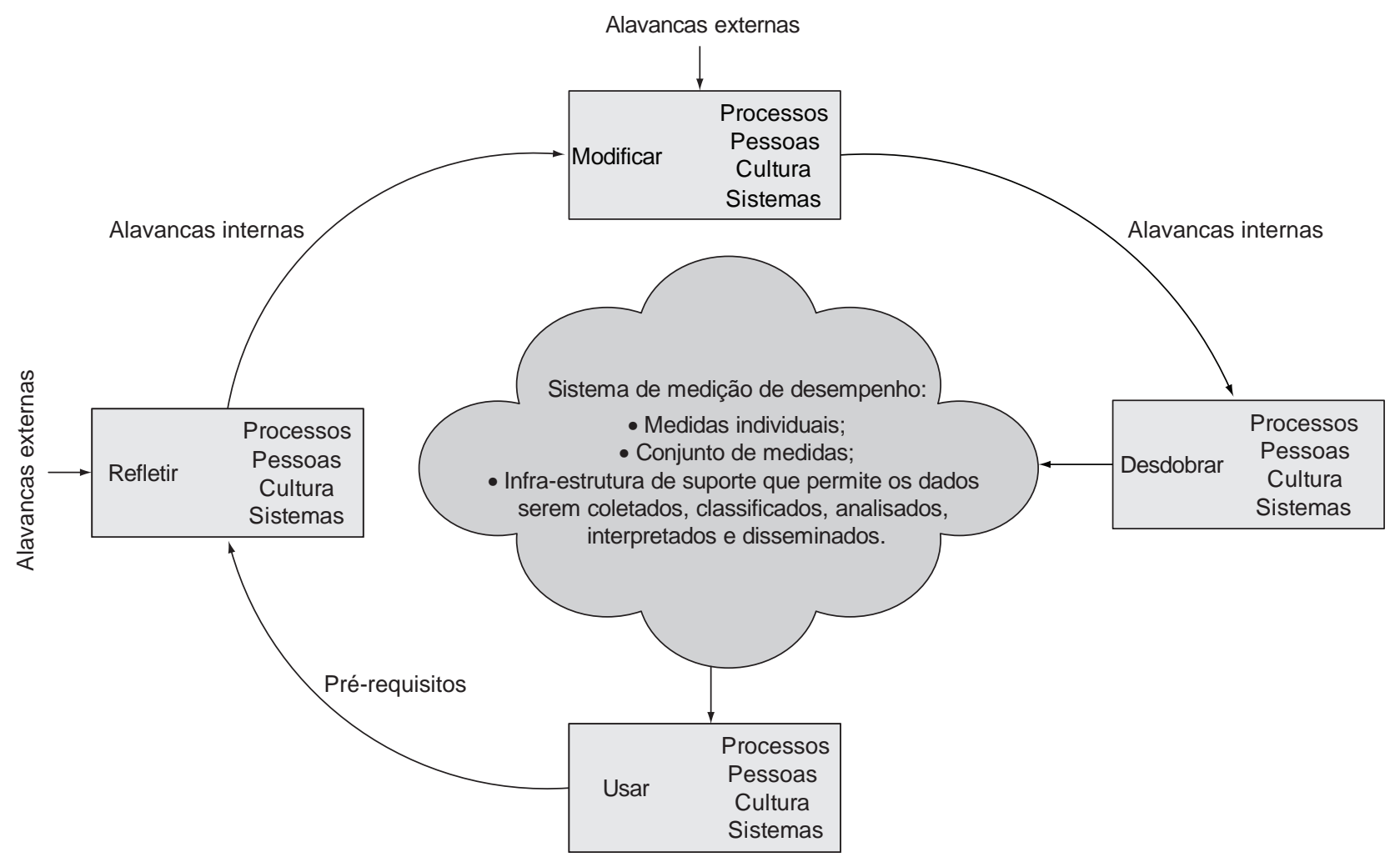

Figura 1. Modelo do processo de evolução dos sistemas de medição de desempenho. (KENNERLEY; NEELY, 2002, p. 1241). 
Primeiramente, o processo apresentado na Figura 1 deve levar em consideração o SMD como o inter-relacionamento de três elementos (medidas individuais, conjunto de medidas e ambiente), proposto por Neely et al. (1995), e o seu uso. Na fase de reflexão, e a partir das alavancas externas, é preciso identificar criticamente se o sistema é realmente apropriado às circunstâncias organizacionais. Posteriormente, na fase de modificação, ele é alterado para se adequar aos requisitos identificados na fase anterior. Finalizando, o SMD previamente modificado é desdobrado para toda a organização para que possa ser usado e gerido de modo a fornecer informações e dados relevantes para os tomadores de decisão.

De modo a relacionar o exposto anteriormente, Martins et al. (2004) afirmam que as forças apresentadas por Waggoner et al. (1999) são externas e internas e podem assumir a função de alavancas (internas e externas) do processo de gestão do SMD proposto por Kennerley e Neely (2002). Desta forma, essa combinação proporciona um caráter evolutivo aos sistemas de medição de desempenho.

Do mesmo modo, a evolução de um sistema de medição de desempenho pode ser impulsionada por uma alavanca externa, como o aumento de competitividade no mercado e a conseqüente adoção de um novo de modelo de gestão ou de práticas desse novo modelo, que requer um uso ou estrutura diferentes da medição de desempenho. Por exemplo, uma organização pode adotar o modelo de gestão pela qualidade total (GQT) do Prêmio Nacional da Qualidade como forma de manter ou aumentar a competitividade. Como o prêmio tem critérios específicos sobre a medição de desempenho, isto requererá o desenvolvimento de novas formas de uso da medição, o que pode desencadear o processo de evolução por meio de uma reflexão, modificação e desdobramento de um novo SMD.

\section{Prêmio Nacional da Qualidade}

Esta seção tem como finalidade apresentar e explanar os aspectos relevantes do modelo de gestão do Prêmio Nacional da Qualidade (PNQ) para a pesquisa realizada. Entretanto, vale ressaltar que poucas referências bibliográficas sobre o assunto foram encontradas. Assim, há a necessidade de pautá-lo em um contexto mais amplo, outros prêmios da qualidade e o modelo de gestão pela qualidade total.

Os prêmios de excelência em qualidade e negócios, que reconhecem o desempenho organizacional de excelência, emergiram como um componente importante de produtividade e de qualidade, fornecendo modelos a partir de conceitos fundamentais da GQT para alcançar o desenvolvimento organizacional e o sucesso do negócio para longo prazo. Assim, o crescimento de prêmios de excelência tem encorajado empresas a adotarem estes modelos de gestão para auto-avaliação organizacional (BIAZZO; BERNARDI, 2003).

Neste sentido, Puay et al. (1998) afirmam que os prêmios da qualidade representam esforços dos países para melhorar a reputação internacional no mercado mundial. Similarmente, Miguel et al. (2004) menciona que os prêmios de excelência em qualidade e negócios, que reconhecem desempenho organizacional de excelência, têm se revelado um componente significativo das estratégias de produtividade e promoção da qualidade em muitos países. Além disso, para Puay et al. (1998), muitas empresas têm relatado que a implementação de modelos de prêmios de excelência não somente melhoram a qualidade, mas também levam a um aumento da participação de mercado, satisfação do cliente, lucratividade, processos, desempenho de fornecedores, moral dos empregados e competitividade.

De maneira a compreender melhor os prêmios da qualidade, há de se considerar os mais importantes no cenário mundial. De acordo com Kholl e Tan (2003), Tan (2002) e Puay et al. (1998), os prêmios de qualidade nacionais mais renomados são: o Deming Prize (Japão); o Malcolm Baldrige National Quality Award (EUA) e o European Quality Award (Europa). No Brasil, o Prêmio Nacional da Qualidade é um reconhecimento da excelência na gestão das organizações, cabendo sua administração à Fundação Prêmio Nacional da Qualidade (FPNQ), uma entidade privada sem fins lucrativos criada em 1991. Desde 2005, essa instituição passou a ser denominada de Fundação Nacional da Qualidade (FNQ).

O modelo de excelência do PNQ é composto dos seguintes critérios de excelência (CE's) (FNQ, 2006; 2007): 1) Liderança; 2) Estratégias e planos; 3) Clientes; 4) Sociedade; 5) Informações e conhecimento; 6) Pessoas; 7) Processos; e 8) Resultados. A Figura 2 ilustra esse modelo e os CE's do PNQ para o ano de 2007.

Os critérios de excelência são fundamentados nos seguintes princípios: pensamento sistêmico; aprendizado organizacional; cultura da inovação; liderança e constância de propósitos; orientação por processos e informações; visão de futuro; geração de valor; valorização das pessoas; conhecimento sobre o cliente e o mercado; desenvolvimento de parcerias e responsabilidade social (FNQ, 2007). O modelo de excelência do PNQ é estabelecido a partir de oito critérios de excelência e 24 itens de excelência (IE's), constituindo um modelo sistêmico de gestão (FPNQ, 2007). A Figura 3 apresenta IE's do PNQ para o ano de 2007.

Os itens de excelência, exceto aqueles do critério de excelência 8 , são atendidos por práticas de gestão da organização. A Figura 4 ilustra, de forma genérica, o diagrama de gestão do PNQ. Existem dois estágios de maturidade das práticas. Um estágio diz respeito ao esta- 
belecimento da gestão da rotina na organização (práticas e padrões) - parte superior da Figura 4. Outro estágio trata da melhoria das práticas e padrões, que no modelo do PNQ é tratado como o aprendizado - parte inferior da mesma figura. Vale assinalar que um dos pilares da GQT

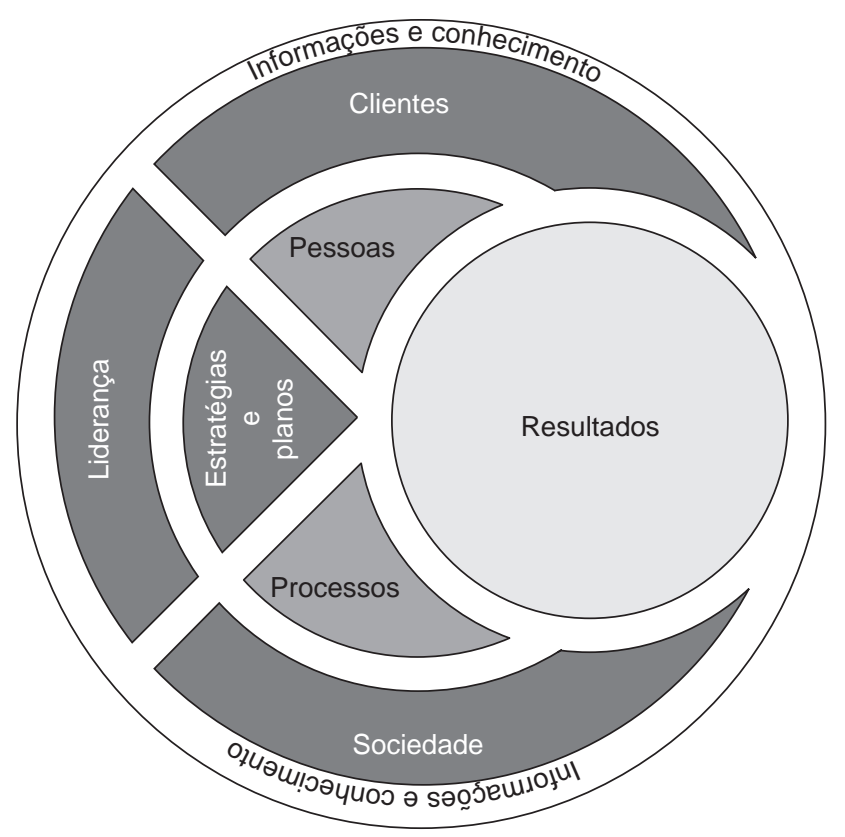

Figura 2. Modelo de excelência ${ }^{\circledR}$ do PNQ. (FNQ, 2007, p. 12). é a gestão da rotina e da melhoria pela aplicação do ciclo PDCA (CAMPOS, 1992).

Para fins da pesquisa realizada, os itens e os critérios mais diretamente relacionados à problemática a ser investigada são: item 1.3 "Análise do desempenho da organização"; critério 2 "Estratégias e planos"; item 5.1 "Informações da organização"; item 5.2 "Informações comparativas"; critério 7 "Processos"; e critério 8 "Resultados". Esses critérios e itens, de uma forma mais direta, tratam da medição de desempenho nos seus vários aspectos, com uma visão sistêmica mencionada por Neely et al. (1995). Vale ressaltar que o tratamento é diferenciado em cada critério ou item relacionado anteriormente.

Neste mesmo contexto, vale destacar a importância do item 2.3 para a adoção de um sistema de medição de desempenho para a organização (FPNQ, 2001). Para tanto, o comitê temático de medição do desempenho global da Fundação para o Prêmio Nacional da Qualidade foi formado em agosto de 1999 por 27 organizações de diversos setores.

Assim, de acordo com FPNQ (2004), as funções primordiais de um sistema de medição de desempenho global são as seguintes: comunicar a estratégia e as prioridades da alta direção e dos gestores; servir como a base para o reconhecimento da dedicação coletiva; analisar problemas estratégicos de forma pró-ativa; apoiar a

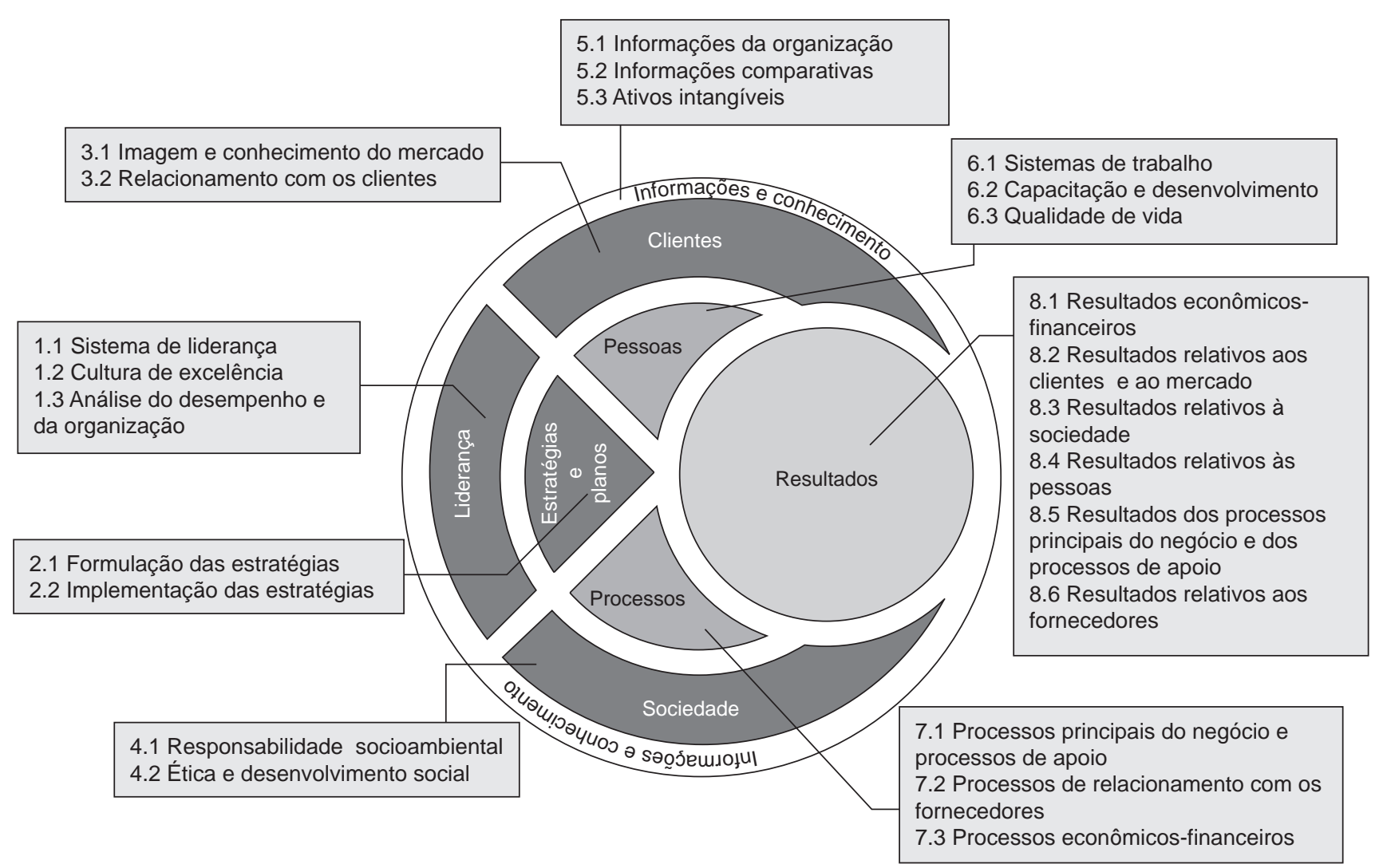

Figura 3. Itens de excelência do PNQ (Adaptado de FNQ, 2007). 


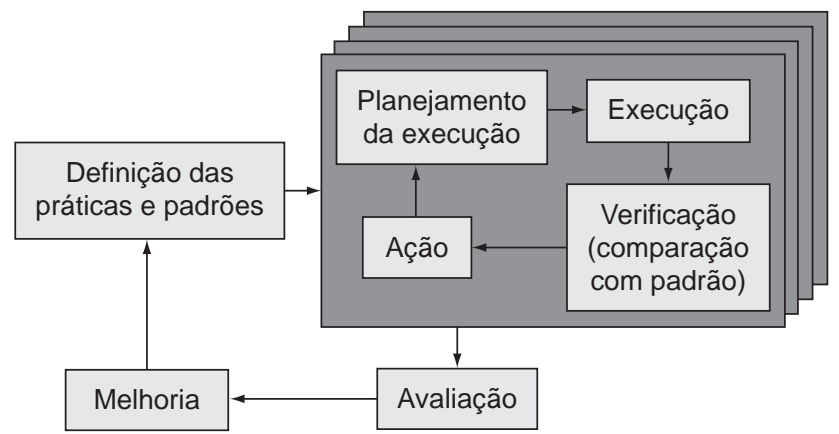

Figura 4. Diagrama de gestão do PNQ (FNQ, 2007, p.15).

tomada de decisão; apoiar a busca de novos caminhos estratégicos para a organização; e apoiar o aprendizado da organização.

Vale salientar que o modelo de gestão do Prêmio Nacional da Qualidade requer certo grau de maturidade em medir o desempenho e usar essa informação para a tomada de decisão organizacional. Desta forma, é relevante estudar empiricamente como as organizações que adotaram o modelo de gestão do PNQ mudaram seus sistemas de medição de desempenho a partir da implementação de novas práticas de gestão.

\section{Método de pesquisa}

O método de pesquisa adotado foi o de estudo de caso explanatório, visto que o objetivo é investigar uma suposta relação causal do modelo de gestão adotado, no caso o do PNQ, e a medição de desempenho. Outro motivo para escolha desse método é a investigação empírica tendo como fonte de informação o ambiente onde essas transformações aconteceram e os atores envolvidos (YIN, 1994). Isto também caracteriza uma abordagem qualitativa em que a perspectiva dos atores envolvidos na problemática é relevante para entendimento do relacionamento causal estabelecido (BRYMAN, 1989).

De forma a permitir comparações cruzadas entre as empresas selecionadas para estudo, optou-se por estudo de caso múltiplo com a finalidade de aumentar e diversificar a quantidade de informações coletadas. Isto permitiu a comparação entre os casos realizados para um melhor entendimento do fenômeno estudado (YIN, 1994). A empresa ganhadora do PNQ foi a unidade de análise investigada, uma vez que nessa organização as práticas de gestão estão consolidadas. Vale também destacar que a FNQ não divulga os nomes das organizações finalistas. Isto impediu a inclusão dessas organizações no rol de casos estudados.

Para o desenvolvimento dos estudos de caso, foram utilizados questionários semi-estruturados para se obter um grau de consistência para: analisar o caso estudado; poder captar perspectivas pessoais das pessoas envolvidas no processo; e, por fim, prover o mínimo de direção para a entrevista. Deste modo, e de acordo com os critérios e itens mais diretamente relacionados à problemática a ser investigada, citados na seção referente ao Prêmio Nacional da Qualidade, buscou-se entrevistar pessoas envolvidas tanto com a adoção do PNQ quanto na medição de desempenho, como:

a) coordenador da adoção do PNQ;

b) pessoas envolvidas na auto-avaliação, principalmente nos itens $1.3,5.1,5.2$ e nos critérios 2,7 e 8 do PNQ;

c) pessoas envolvidas com a elaboração do relatório de gestão dos itens $1.3,5.1,5.2$ e critérios 2,7 e 8 do PNQ; e

d) pessoas responsáveis pelas mudanças nas práticas de medição de desempenho da organização por conta da adoção do PNQ.

Para analisar as mudanças no sistema de medição de desempenho após a adoção do PNQ, foram consideradas as informações referentes tanto às práticas de gestão utilizadas antes e depois da adoção do PNQ quanto ao histórico de gestão da qualidade das empresas estudadas. Além disso, foi feita uma análise de cada caso e outra entre os casos.

\section{Resultados da pesquisa de campo}

Seguindo os critérios estabelecidos na seção anterior, um estudo de caso múltiplo foi realizado em três empresas ganhadoras do Prêmio Nacional da Qualidade em 2005: Serasa, CPFL Paulista e Suzano Petroquímica. Os entrevistados, em cada empresa estudada, foram:

a) Serasa: assessor líder da qualidade e duas pessoas da assessoria da qualidade;

b) CPFL paulista: Assessor de gestão da qualidade, engenheiro de planejamento da qualidade e a analista da qualidade; e

c) Suzano Petroquímica: analista da gerência de desenvolvimento de processos e projetos, gerente de saúde, segurança, conservação ambiental e qualidade, coordenador de TI, e coordenador de tecnologia.

Entretanto, vale mencionar que as pessoas entrevistadas muitas vezes estavam envolvidas e eram responsáveis tanto com a adoção do PNQ quanto com o sistema de medição de desempenho.

As principais características das empresas estudadas estão sumarizadas na Tabela 1. De acordo com esses dados, pode-se notar que as empresas estudadas são de capital nacional, sendo pertencentes a três diferentes setores industriais: prestação de serviços, distribuição de energia elétrica e petroquímica. Com relação à adoção do modelo do PNQ, a Serasa começou a adoção dos CE's em 1992 , enquanto as outras o fizeram a partir de 2000. Isto 
Tabela 1. Principais características das empresas estudadas. Fonte: Empresas estudadas.

\begin{tabular}{|c|c|c|c|}
\hline & Serasa & CPFL Paulista & Suzano Petroquímica \\
\hline Capital & Nacional & Nacional & Nacional \\
\hline Setor industrial & Prestação de serviços & Distribuição de energia elétrica & Petroquímico \\
\hline Porte & Grande & Grande & Médio \\
\hline Principais produtos & $\begin{array}{l}\text { Análises e informações para } \\
\text { decisões de crédito e apoio a } \\
\text { negócios }\end{array}$ & Energia elétrica & Polipropileno \\
\hline Número de funcionários & $\begin{array}{l}2073 \text { funcionários, } 91 \text { terceiri- } \\
\text { zados e } 40 \text { estagiários }\end{array}$ & $\begin{array}{l}3018 \text { funcionários, } 87 \text { prestado- } \\
\text { res de serviços e } 55 \text { estagiários }\end{array}$ & $\begin{array}{l}465 \text { empregados, } 130 \text { terceiri- } \\
\text { zados e } 10 \text { estagiários }\end{array}$ \\
\hline Início da adoção do PNQ & 1992 & 2002 & 2000 \\
\hline Histórico da premiação do PNQ & $\begin{array}{l}\text { Finalista em } 1994 \text { e vencedora } \\
\text { em } 1995,2000 \text { e } 2005\end{array}$ & $\begin{array}{l}\text { Finalista em } 2004 \text { e vencedora } \\
\text { em } 2005\end{array}$ & $\begin{array}{l}\text { Finalista em } 2003 \text { e vencedo- } \\
\text { ra em } 2005\end{array}$ \\
\hline Sistema de medição de desempenho & $\begin{array}{l}\text { Hierarquia estratégica de } \\
\text { indicadores }\end{array}$ & Baseado no Balanced Scorecard & Balanced Scorecard \\
\hline
\end{tabular}

pode sugerir que os momentos diferentes de adoção do modelo do PNQ nas empresas estudadas podem acarretar em alterações no sistema de medição de desempenho das empresas.

Do mesmo modo, para o processo de adoção do modelo de gestão do PNQ pelas empresas estudadas, é relevante enfatizar dois pontos. Primeiro, a estratégia de adoção do modelo foi top-down para as três empresas estudadas. Segundo, em todas houve a necessidade do auxílio de consultoria externa. Isto demonstra a abrangência da mudança e o grau de dificuldade.

No sentido de traçar a trajetória da evolução da gestão da qualidade das empresas na implementação do modelo, alguns pontos devem ser destacados:

a) a Serasa buscou a certificação ISO 9001 em 1997, após a sua adequação de gestão aos critérios do PNQ;

b) o sistema de gestão da CPFL Paulista e os seus principais processos possuem as seguintes certificações: ISO 9001, ISO 14001, OHSAS 18001 e AS 8000 antes da adoção do PNQ; e

c) a Suzano certificou-se nas normas ISO 9001, QS 9000, ISO 14001, OHSAS 18001 antes da adoção do PNQ.

Assim, pôde ser percebido que as trajetórias da CPFL e da Suzano Petroquímica parecem corroborar com o sugerido por Oliveira e Martins (2005). Esses autores afirmam, a partir de uma revisão bibliográfica acerca da ISO 9001 e PNQ, que o caminho natural após a adequação e consolidação do modelo de gestão da qualidade da ISO 9001 pode ser a adoção do modelo de excelência de prêmios da qualidade. Vale lembrar que Martins e Miranda (2005) observaram, a partir de estudos de caso, que a implementação do modelo da ISO 9001 requereu mudanças na medição de desempenho das empresas estudadas por eles.

No entanto, nota-se que a evolução de gestão da qualidade na Serasa seguiu uma direção contrária às outras organizações, considerando que, como afirmado pelo assessor da qualidade, a empresa considera o PNQ uma forma de auto-avaliação e não um modelo de gestão. Vale observar que essa empresa foi ganhadora do Prêmio Nacional da Qualidade nos anos de 1995, 2000 e 2005.

A CPFL Paulista, para ganhar o Prêmio Nacional da Qualidade, teve ações ao longo da sua trajetória, tais como: envolvimento da alta direção; disseminação dos fundamentos e critérios de excelência para a organização; filiação a FNQ e participação nos seminários e cursos de capacitação; formação interna de colaboradores: representantes de áreas por critério; análise crítica do relatório de avaliação; proposição de planos de ação para implementar as oportunidades de melhorias sinalizadas nos relatórios de avaliação da Associação Brasileira de Distribuição de Energia Elétrica (ABRADEE) e da FNQ.

Para a Suzano Petroquímica, vale destacar alguns pontos referentes à sua premiação, como vontade e compromisso da alta administração, busca por entendimento dos critérios em relação aos seus processos de gestão, disseminação e implantação de mecanismos de controle e aprendizado, e por fim, avaliações das práticas de gestão.

Pode ser percebido que as três empresas estudadas, mesmo tendo trajetórias diferentes para a adoção e premiação do PNQ, conseguiram mobilizar e incentivar a força de trabalho para a compreensão dos critérios de excelência na implementação de práticas de gestão adequadas aos itens de excelência.

Com relação às mudanças ocorridas na medição de desempenho das empresas estudadas, algumas particularidades podem ser observadas, tais como: 1) poucas alterações nas práticas de gestão na Serasa; 2) modificações e incorporação de práticas de gestão na CPFL Paulista; e 3) mudanças mais substanciais na Suzano Petroquímica. Assim, observa-se que os efeitos do modelo de gestão PNQ na medição de desempenho parecem estar 
relacionados com o histórico de gestão da qualidade da empresa para a adoção do PNQ.

Isto pode explicar as modificações sutis na Serasa, uma vez que a empresa havia se adequado anteriormente ao modelo do Prêmio Nacional da Qualidade - a primeira vez ocorreu em 1992. Isto também pode explicar a afirmação do assessor da qualidade entrevistado, citado anteriormente, que o modelo do PNQ é utilizado como uma forma de auto-avaliação. Assim, para essa empresa, o modelo de gestão baseado no PNQ consolidado parece ter ocasionado poucas mudanças na medição de desempenho.

Já na CPFL Paulista, foi observada a existência tanto da modificação das práticas de gestão quanto a incorporação de novas práticas. A empresa em questão desenvolveu certo número de práticas antes da adoção do modelo de gestão do PNQ, pois a sua gestão da qualidade foi estruturada em 2000, por meio da criação da assessoria de gestão da qualidade, do lançamento do programa selo qualidade CPFL, do surgimento da avaliação das práticas de gestão pela ABRADEE e do Prêmio 5S.

Por fim, na Suzano Petroquímica, foi verificado que ocorreram diversas mudanças tanto no sentido de reorganização e alinhamento estratégico quanto no sentido das práticas de gestão para a medição de desempenho. Isto acarretou mudanças no sistema de medição de desempenho da empresa como um todo.

Com o intuito de gerar subsídios mais concretos para essa discussão, a Tabela 2 sintetiza as principais práticas de gestão nas empresas estudadas, antes e depois da adoção do modelo de excelência do Prêmio Nacional da Qualidade.

No caso da Serasa, uma mudança relevante da organização para se adequar ao modelo de gestão do PNQ, que pode ser destacada, é a estruturação dos sistemas de informações gerenciais para o processo de seleção, gestão e disseminação das informações sobre os indicadores de desempenho. Vale recordar que essa empresa não necessitou realizar mudanças profundas em seu sistema de gestão para se concorrer ao prêmio. Contudo, vale enfatizar que a empresa se empenhou em buscar o crescimento contínuo por meio de avaliações e auditorias internas com o intuito de propor melhorias e planos de ação, bem como treinamentos com os funcionários para estabelecer competências internas nas práticas de gestão implementadas.

Na CPFL Paulista, foi observado que os efeitos da adoção do modelo de gestão do PNQ não foram tão substanciais pela existência da gestão da qualidade na empresa. As práticas de gestão já estabelecidas não foram modificadas, mas sim melhoradas, e outras foram incorporadas com o intuito da empresa de adequá-las ao modelo de gestão do PNQ.
Deste modo, após a adoção de tal modelo, destaca-se o surgimento de algumas práticas com finalidades diferentes para atender o diagrama de gestão do PNQ (Figura 4), tais como:

a) mecanismos de controle: auditoria interna do SGI, auditoria externa do SGI, reunião da diretoria, auditoria interna, avaliação das estações avançadas e controle gerencial; e

b) mecanismos de aprendizado: avaliação do PNQ, avaliação da ABRADEE, reunião de análise crítica do sistema de gestão integrado, workshop de análise crítica do sistema de gestão integrado, análise crítica das auditorias internas e reunião da diretoria.

Com relação às práticas de gestão da Suzano Petroquímica, as alterações ocorreram no âmbito do planejamento estratégico e do sistema de medição de desempenho. Primeiro, destaca-se a implantação do Balanced Scorecard que requereu a modificação na estruturação e sistematização do planejamento estratégico pela alteração na utilização das informações provenientes dos indicadores de desempenho. No entanto, é necessário apontar a importância do gerenciamento do desempenho juntamente com a utilização do fator $\mathrm{ABC}$ para a estruturação e sistematização do BSC.

Desta forma, pode ser percebido o surgimento de algumas práticas de gestão na Suzano Petroquímica em decorrência da implantação do BSC, como: painel de gestão estratégica (PGE), sistema de faróis do painel de bordo, mapa estratégico, e reuniões no comitê de gestão estratégica e de processo.

No âmbito do sistema de medição de desempenho para fornecer suporte à gestão, item de excelência 2.3 do PNQ (Figura 3), na Serasa foi desenvolvido um modelo de sistema de medição de desempenho, a hierarquia estratégica de indicadores, que está alinhado ao conceito do Balanced Scorecard, pois tem por objetivo integrar os indicadores organizacionais e permitir o equilíbrio entre as perspectivas estratégicas e operacionais. Esse modelo ainda enfatiza a correlação entre os indicadores, algo que não é muito claro no BSC.

Do mesmo modo, na CPFL foi observado um sistema de medição de desempenho baseado no BSC. Entretanto, aparentemente a maioria dos seus indicadores para a tomada de decisão da alta administração é essencialmente de caráter financeiro.

Já na Suzano, em 2003, foi adotado o Balanced Scorecard com o intuito de reorganizar, sistematizar, alinhar, disseminar e comparar as medidas de desempenho. Assim, pode ser notado que a implantação de tal sistema de medição parece ter sido impulsionada pelo modelo de gestão do PNQ, como sugerido por FPNQ (2004).

Desta forma, pode ser notado que as empresas estudadas desenvolveram ou adotaram SMD's baseados na proposta de Kaplan e Norton (1992, 1997), que 
Tabela 2. Principais práticas de gestão das empresas estudadas. Fonte: Empresas estudadas.

\begin{tabular}{|c|c|c|}
\hline \multicolumn{3}{|c|}{ Práticas de gestão } \\
\hline & Antes da adoção do PNQ & Depois da adoção do PNQ \\
\hline Serasa & $\begin{array}{l}\text { Processo da qualidade Serasa; } \\
\text { Reuniões do comitê diretivo; } \\
\text { Relatório anual geral; } \\
\text { Rede de informação para gestão; e } \\
\text { Gestão da tecnologia e das telecomuni- } \\
\text { cações direcionada para capacidade de } \\
\text { processamento e armazenamento de dados. }\end{array}$ & $\begin{array}{l}\text { Hierarquia estratégica de indicadores; } \\
\text { Prêmios internos das diretorias; } \\
\text { Treinamentos in company dos critérios de excelência do PNQ } \\
\text { com funcionários; } \\
\text { Hierarquia estratégica de indicadores; e } \\
\text { Sistemas de informações gerenciais (MIS). }\end{array}$ \\
\hline CPFL Paulista & $\begin{array}{l}\text { Programa de qualidade total; } \\
\text { Sistema de gestão integrado; } \\
\text { Plano Millenium; } \\
\text { Sistema Millenium; } e \\
\text { Site do conselho de administração. }\end{array}$ & $\begin{array}{l}\text { Painel de controle; } \\
\text { Relatório de gestão; } \\
\text { Auditoria interna do SGI; } \\
\text { Auditoria externa do SGI; } \\
\text { Reunião da diretoria; } \\
\text { Auditoria interna (avaliação dos processos e riscos na opera- } \\
\text { ção); } \\
\text { Avaliação das estações avançadas; } \\
\text { Controle gerencial; } \\
\text { Avaliação do PNQ; } \\
\text { Avaliação da ABRADEE } \\
\text { Reunião de análise crítica do sistema de gestão integrado; } \\
\text { Workshop de análise crítica do sistema de gestão integrado; } \\
\text { Análise crítica das auditorias internas; } \\
\text { Reunião da diretoria para o acompanhamento mensal da } \\
\text { implementação de inovações e/ou melhorias das práticas e } \\
\text { padrões de trabalho; } \\
\text { Sistema Prometa; e } \\
\text { Sistema notes. }\end{array}$ \\
\hline Suzano Petroquímica & $\begin{array}{l}\text { Reunião de processo; } \\
\text { Reunião interna; } \\
\text { Ferramentas da qualidade (fluxograma, } \\
\text { diagrama de causa e efeito, análise de } \\
\text { pareto, histograma, estratificação, diagrama } \\
\text { de dispersão, folhas de coletas de dados e } \\
\text { verificação, gráficos de controle, gráficos } \\
\text { demonstrativos e brainstorming); } \\
\text { Documentação de todos os processos; } \\
\text { Programa de atuação responsável; } \\
\text { Sistema integrado de gestão; } \\
\text { Relatório de gestão; } \\
\text { Gerenciamento de desempenho; } \\
\text { Fator ABC; e } \\
\text { ERP. }\end{array}$ & $\begin{array}{l}\text { Painel de gestão estratégica (PGE); } \\
\text { Sistema de faróis do painel de bordo; } \\
\text { Processo de formulação das estratégias; } \\
\text { Execução do businnes planning; } \\
\text { Gestão de pessoas e iniciativas estratégicas; } \\
\text { KISS (keep in short single); } \\
\text { Reunião de vídeo conferência; } \\
\text { Tótens de divulgação; } \\
\text { Balanced Scorecard; } \\
\text { Software Hypireon; } \\
\text { EIS (executive inteligence systems); } \\
\text { Mapa estratégico; } \\
\text { Reunião de avaliação de desempenho; } \\
\text { Reuniões no comitê de gestão estratégica e de processo; } \\
\text { Intranet como meio de comunicação das estratégias e indica- } \\
\text { dores de desempenho; } \\
\text { Dashboard; } \\
\text { Prática de benchmarking; } \\
\text { Processo de estabelecimento dos padrões de trabalho; e } \\
\text { Pesquisa de eficiência interna. }\end{array}$ \\
\hline
\end{tabular}

contempla tanto as medidas de desempenho financeiras quanto as não-financeiras. Além do mais, considerando que FPNQ (2004) recomenda a utilização de diferentes perspectivas para o estabelecimento do mapa estratégico, é possível apontar que as empresas estudadas estruturaram seus sistemas de medição de desempenho de forma a abranger as necessidades de diferentes áreas de negócio e partes interessadas, não se restringindo às perspectivas propostas por Kaplan e Norton (1992, 1997).
Para as medidas de desempenho individuais, na Serasa, segundo os entrevistados, a criação das mesmas é de responsabilidade de cada funcionário, que julga a sua necessidade em relação ao trabalho e função que exerce. Para tanto, essa empresa possui uma grande quantidade de medidas de desempenho individuais que são criadas, monitoradas e utilizadas de acordo com a necessidade dos usuários. 
Para os indicadores de desempenho, a CPFL Paulista criou o painel de controle para integrar as informações dos software de gestão em uma única base de dados, bem como acompanhar os indicadores de desempenho, os resultados e as avaliações críticas de desempenho. Assim, pode-se perceber que os indicadores de desempenho, as informações e as decisões parecem estar alinhados e coesos em todos os níveis hierárquicos da organização.

A Suzano Petroquímica utiliza, de forma a desdobrar e controlar as medidas de desempenho, o software Hypireon que dá suporte ao acompanhamento e controle dos indicadores de desempenho bem como a implementação de ações de melhoria. Ainda, vale destacar que esse software foi adquirido para oferecer suporte ao desenvolvimento dos mapas estratégicos do BSC.

As medidas de desempenho e os SMD's observados na pesquisa de campo parecem convergir para as definições sugeridas por Neely et al. (1995), Neely (1998), Kennerley et al. (2003) e Franco-Santos et al. (2004), em que o SMD é formado por medidas individuais que quantificam o impacto de ações específicas, um conjunto de medidas combinadas e relacionadas para avaliar o desempenho organizacional, e uma sistemática de medição de desempenho que permite que dados de desempenho sejam coletados, classificados, analisados, interpretados e disseminados para o uso gerencial. Foi observado uso nos três níveis hierárquicos: estratégico, tático e operacional.

No sentido de poder oferecer suporte ao uso da medição de desempenho, a Serasa possui uma rede de informações para a gestão que tem por objetivo integrar e relacionar as informações sobre os indicadores de desempenho em todos os níveis hierárquicos. Contudo, deve ser destacado que essa empresa não possui um sistema entreprise resources planning (ERP). Diferentemente dessa empresa, a CPFL Paulista dispõe de um sistema de gestão único do tipo ERP, que possibilita a integração dos sistemas de informação para gestão para suporte à tomada de decisão.

Do mesmo modo, a Suzano Petroquímica possui um sistema ERP, que possibilita a integração das informações. Neste mesmo sentido, são desenvolvidos executive information systems (EIS's), que visam atender às necessidades específicas dos usuários para tomada de decisão. Eles são desenvolvidos pela área de informática da empresa. Os EIS's para suprimentos e vendas foram apresentados para os pesquisadores.

Considerando os sistemas de informação utilizados pelas empresas estudadas, pode ser notado que nos processos de gestão de desempenho analisados há sistemas de informação que buscam integrar todas as informações de desempenho relevantes (BITITCI et al., 1997). O seu uso parece facilitar as tarefas do sistema de medição de desempenho por meio do armazenamento e comunicação dos dados de desempenho em uma plataforma central.

Em decorrência da tecnologia utilizada, foi notado que as empresas estudadas buscam a obtenção e o acompanhamento das informações sobre os indicadores de desempenho em várias áreas da empresa, em diferentes níveis hierárquicos e para usos diversos. De tal modo, indica-se a notável importância do uso das informações sobre o desempenho para guiar o processo de desenvolvimento de sistemas de medição de desempenho, como proposto por Martins (2002).

Por fim, vale destacar as vantagens e as desvantagens da adoção do PNQ para as práticas de gestão de medição de desempenho. Assim, pode ser ressaltada, para as três empresas estudadas, a estruturação, a simplificação, a disseminação e os entendimentos das práticas de medição de desempenho.

\section{Conclusões}

De uma forma geral, a adoção do modelo de excelência do PNQ, nas empresas estudadas, resultou em impactos distintos nos diferentes elementos de um sistema de medição de desempenho. Isto está associado ao fato de, ao adotar o modelo, as práticas de gestão das organizações estudadas estarem em diferentes estágios, devido ao histórico de gestão da qualidade das empresas, indo desde a inexistência até a prática madura.

Um exemplo disto é a Serasa. Por ser vencedora em duas oportunidades anteriores e estar utilizando o modelo do PNQ como uma forma de auto-avaliação há algum tempo, o impacto da adoção do modelo no SMD não é tão claro à primeira vista. Isto ocorre porque a maioria das práticas de gestão da organização está em fase de aprendizado, conforme diagrama de gestão da Figura 4, em que os impactos não são visíveis assim. Por outro lado, na Suzano foi mais fácil observar o impacto de práticas de gestão recém implementadas, pois os entrevistados ainda não as consideravam como parte normal de suas atividades.

Tomando como elementos as medidas individuais, a estrutura do sistema de medição de desempenho e a infra-estrutura de suporte, é possível examinar com mais detalhes os efeitos da adoção do PNQ nos SMD's das empresas pesquisadas.

As mudanças nas medidas individuais não foram visíveis. Isto se deve, principalmente, à maturidade da gestão da qualidade das empresas estudadas. Vale destacar que podem ter ocorrido mudanças na Serasa e na Suzano, uma vez que nessas organizações foi observada forte ênfase em prover os tomadores de decisão com informações cuja fonte seriam as medidas individuais. Em ambos os casos, o uso, respectivamente, do MIS e do EIS podem ter induzido a criação de novas medidas individuais. 
Todavia, a observação dessa ocorrência não foi facilitada pelo uso do método de estudo de caso. Tais mudanças podem ser associadas ao critério de excelência "Informação e conhecimento".

As mudanças na estrutura do sistema de medição de desempenho foram observadas mais nitidamente na Serasa, hierarquia estratégica de indicadores, e na Suzano, Balanced Scorecard. O sistema da Serasa tem como diferencial do BSC de Kaplan e Norton (1992; 1997) a correlação estatística entre as medidas individuais. Vale assinalar que na CPFL foi observada uma estrutura de SMD semelhante ao BSC, mas que os entrevistados admitiram não ser tal sistema. Tais modificações podem ser atribuídas ao critério de excelência "Estratégia e planos".

As mudanças na infra-estrutura de suporte foram observadas com maior ênfase na forma de analisar, interpretar e disseminar as informações sobre desempenho. O maior impacto observado nessas atividades foi a aplicação de software e tecnologia de informação. O MIS e EIS observados, respectivamente, na Serasa e Suzano contribuem muito para essas atividades, principalmente por levarem ao usuário um meio informatizado para fazer análises das diversas formas e para diferentes objetivos e tomadas de decisão.

Outras formas de disseminação foram também observadas. $\mathrm{Na}$ CPFL, o painel de controle unifica numa plataforma diversas informações sobre desempenho num ambiente gráfico, via rede de computadores, para diversos usuários. Algo semelhante foi observado na Serasa e na Suzano. Nesta última, também foram observados totens em alguns setores da empresa com gráficos e informações sobre diversas medidas de desempenho. Isto se deve ao critério de excelência "Informação e conhecimento".

Ainda sobre infra-estrutura, foram observadas práticas de gestão que causaram mudanças nas formas de analisar e interpretar os dados sobre desempenho. Na CPFL, o Sistema Prometa associa iniciativas e projetos, que são desdobrados do planejamento estratégico, servindo de base para acompanhamento nas reuniões mensais. Tal sistema é utilizado em conjunto com o painel de controle. Já na Suzano, o Fator ABC (em que C são objetivos e metas da alta administração, B são metas dos processos relacionadas às metas $\mathrm{C}$, e $\mathrm{A}$ são as metas individuais relacionadas às metas $\mathrm{B}$ ) juntamente com o mapa estratégico do BSC facilita o desdobramento e o controle de objetivos e metas estratégicos. Isto está relacionado ao item de excelência "Análise do desempenho da organização".

Vale destacar aqui o papel importante que as soluções de tecnologia de informação exercem nas mudanças observadas com relação à infra-estrutura de suporte dos SMD's das empresas estudadas. Em todos os casos, foram observadas as mais diversas aplicações e a sua importância para facilitarem o uso dos sistemas de medição de desempenho por vários colaboradores em diversos níveis hierárquicos. Isto merece mais atenção dos pesquisadores da área, uma vez que a área de tecnologia de informação está sempre apresentando inovações que podem melhorar a infra-estrutura dos SMD's.

Por fim, os dados do estudo de caso múltiplo confirmaram o que foi levantado na pesquisa bibliográfica, em que a adoção do modelo do PNQ pode agir como um vetor de mudanças na medição de desempenho. Isto acontece de duas formas, em concordância com a proposta de Kennerley e Neely (2002), ilustrada na Figura 1.

Uma forma é como uma alavanca externa. Isto ocorre por meio dos critérios de excelência "estratégia e planos" e "informação e conhecimento", nos momentos de refletir (auto-avaliação frente ao modelo) e modificar o SMD, conforme a proposta de Kennerly e Neely (2002). Isto afeta principalmente a estrutura do modelo de medição de desempenho, adoção do BSC nos casos estudados, e nas medidas de desempenho.

Outra forma é como alavanca interna. Isto acontece por meio da implementação de novas práticas de gestão e modificação de outras existentes, e também pela estruturação do Diagrama de Gestão, ilustrado na Figura 4, nos momentos de refletir e modificar o SMD, conforme a proposta de Kennerly e Neely (2002). No entanto, tais alterações podem ser sutis, de forma que sua compreensão necessite de estudos mais aprofundados nas empresas estudadas. Isto pode ser um possível encaminhamento futuro da pesquisa realizada.

Por fim, pode-se concluir, com base nos casos estudados, que a adoção do modelo de excelência do PNQ em grau diferente acabou exercendo um papel importante na evolução da medição de desempenho. 


\title{
Effects of the adoption of a national quality award on the measurement of performance: case studies in award-winning companies
}

\begin{abstract}
This paper presents the empirical findings of a multiple case study about the effects of the adoption of the National Quality Award (NQA) on performance measurement. The three companies of this study were winners of the 2005 edition of the award. The main findings indicate that the Excellence Model of the NQA plays an important role as an external and internal lever in the evolution of performance measurements in the companies in question. The excellence criteria of "strategy and plans" and "information and knowledge" exert external leverage, while new or improved management practices exert internal leverage in the stages of reflection, modification and deployment of performance measurement. The changes in performance measurement systems were found to occur in different degrees in the companies of this study.
\end{abstract}

Keywords: Performance measurement. Brazilian national quality award. Performance measurement systems. Excellence model.

\section{Referências bibliográficas}

ATKINSON, A. A.; WATERHOUSE, J. H.; WELlS, R. B. A stakeholder approach to strategic performance measurement. Management Sloan Review, Cambridge, v. 38, n. 3, p. 25-37, 1997.

BIAZZO, S.; BERNARDI, G. Organisational self-assessment options A classification and a conceptual map for SMEs. International Journal of Quality \& Reliability Management, Bradford, v. 20, n. 8, p. 881-900, 2003.

BITITCI, U. S.; CARRIE, A. S.; McDEVITT, L. Integrated performance measurement systems - a development guide. International Journal of Operations \& Production, Bradford, v. 17 , n. 5, p. 552-534, 1997.

BRYMAN, A. Research methods and organization studies. London: Routledge, 1989. 283p.

CAMPOS, V. F. TQC: controle da qualidade total (no estilo japonês). 5 ed. Belo Horizonte: Fundação Christiano Ottoni, 1992. 229p.

CROSS, K. F.; LYNCH, R. L. Managing the corporate warriors. Quality Progress, Milwaukee, v. 23, n. 4, p. 54-59, 1990.

FPNQ - Fundação Prêmio Nacional da Qualidade. Relatório do Comitê Temático: Planejamento do sistema de medição do desempenho global. São Paulo: FPNQ, 2001.

FPNQ - Fundação Prêmio Nacional da Qualidade. Relatório do Comitê Temático: Planejamento do sistema de medição do desempenho global. 2.ed. São Paulo: FPNQ, 2004.

FNQ - Fundação Nacional da Qualidade. Critérios de excelência. São Paulo: FNQ, 2006.

FNQ - Fundação Prêmio da Qualidade. Critérios de excelência. São Paulo: FNQ, 2007.

FRANCO-SANTOS, M.; MARR, B.; MARTINEZ, V.; GRAY, D.; ADAMS, C.; MICHELI, P.; BOURNE, M.; KENNERLEY, M.; MASON, S.; NEELY, A. Towards a definition of a business performance measurement system. In: The Fourth International Conference on Performance Measurement and Management, PMA, Edinburgh, UK, 2004. Proceedings... Bedfordshire: Cranfield Business School/Cranfield University, 2004, p. 395-402.
HRONEC, S. M. Sinais Vitais. São Paulo: Makron Books, 1994. $240 \mathrm{p}$.

KAPLAN, R. S.; NORTON, D. P. The Balanced Scorecard measures that drive performance. Harvard Business Review, New York, v. 70, n. 1, p. 71-79, jan./feb. 1992.

KAPLAN, R, S.; NORTON, D. P. Estratégia em ação - Balanced Scorecard. Tradução de Luiz Euclydes Trindade Frazão Filho. Rio de Janeiro: Campus, 1997. 344p.

KAYDOS, W. Measuring, managing and maximizing performance. Productivity Press. Portland: Oregon, 1991. $259 \mathrm{p}$.

KEEGAN, D. P.; EILER, R. G.; JONES, C. R. Are your performance measures obsolete? Management Accounting, New York, v. 70, n. 1, p. 45-50, 1989.

KENNERLEY, M.; NEELY, A.; ADAMS, C. Survival of the fittest: measuring performance in a changing business environment. Measuring Business Excellence, Bradford, v. 7, n. 4, p. 37-43, 2003.

KENNERLEY, M.; NEELY, A. A framework of the factor affecting the evolution of performance measurement systems. International Journal of Operations \& Production Management, Bradford, v. 22, n. 11, p. 1222-1245, 2002.

KHOLL, H. H.; TAN, K. C. Managing for quality in the USA and Japan: differences between the MBNQA, DP and JQA. The TQM Magazine, West Yorkshire, v. 15, n. 1, p. 14-24, 2003.

KUENG, P.; MEIER, A.; WETTSTEIN, T. Computer-based performance measurement in SMEs: Is there any option? Switzerland: University of Fribourg, Institute of Informatics. Internal Working Paper, n. 00-11, p. 1-15, 2000.

LEBAS, M. J. Performance measurement and performance management. International Journal of Production Economics, Amsterdam, v. 41, n.1-3, p. 23-35, 1995.

MARTINS, R. A.; COSTA NETO, P. L. O. Indicadores de desempenho para a gestão da qualidade total: Uma proposta de sistematização. Gestão \& Produção, São Carlos, v. 5, n. 3, p. 298-311, 1998. 
MARTINS, R. A.; MERGUlhaO, R. C.; CANEVAROLlO, M. E. A. P. Uma investigação empírica sobre a dinâmica da medição de desempenho. In: SIMPEP, 13, 2005, Bauru, SP. Anais... Bauru: UNESP, 2005.

MARTINS, R. A.; MIRANDA, R. A. M. Factors affecting the support of performance measurement to continuous improvement activities. In: INTERNATIONAL CONTINUOUS INNOVATION NETWORK (CINet) Conference, Brighton, UK, 6, 2005. Proceedings... Brighton: CENTRIM/CINet, 2005.

MARTINS, R. A. The use of performance measurement information as a driver designing a performance measurement system. In: The Third International Conference on Performance Measurement and Management, PMA, 2002, Boston - MA, USA. Proceedings... Boston, 2002, p. 371-378.

MARTINS, R. A. Sistemas de medição de desempenho: um modelo para a estruturação do uso. São Paulo, 1998. 248p. Doutorado - (Engenharia de Produção), Escola Politécnica da Universidade de São Paulo - USP.

MASKELL, B. H. Performance measurement for world class manufacturing: A model for American Companies. Portland, Oregon: Productivity Press, 1991. 408p.

McNAIR, C. J.; LYNCH, R. L.; CROSS, K. F. Do Financial and nonfinancial performance measures have to agree? Management Accounting, New York, v. 72, n. 5, p. 28-35, 1990.

MIGUEL, P. A. C. A report on comparing worldwide quality and business excellence awards - part 1: systems of operations, core values and assessment criteria. Santa Bárbara D'Oeste: UNIMEP, 2004. (Relatório de pesquisa)

MIGUEL, P. A. C.; MORINI, C.; PIRES, S. R. I. An Application Case of the Brazilian National Quality Award. TQM Magazine, West Yorkshire, v. 16, n. 3, p.186-193, 2004b.
NEELY, A. Measuring Business Performance. London: The Economist Books Ltd., 1998. 208p.

NEELY, A.; ADAMS, C.; CROWE, P. The performance prism in practice. Measuring Business Excellence, Bradford, v. 5, n. 2, p. 6-12, 2001

NEELY, A.; GREGORY, M.; PLATTS, K. Performance measurement system design - A literature review and research agenda. International Journal of Operations \& Production Management, Bradford, v. 15, n. 4, p. 80-116, 1995.

OLIVEIRA, G. T.; MARTINS, R. A. ISO 9001 como base para a implementação do prêmio nacional da qualidade. In: SIMPEP, 12, 2005, Bauru, SP. Anais... Bauru: UNESP, 2005.

PUAY, S. H.; TAN, K. C.; XIE, M.; GOH, T. N. A comparative study of nine national quality awards. The TQM Magazine, West Yorkshire, v. 10, n. 1, p. 30-39, 1998.

TAN, K. C. A comparative study of 16 national quality awards. The TQM Magazine, West Yorkshire, v. 14, n. 4, p. 165-171, 2002.

WAGGONER, D. B.; NEELY, A. D.; KENNERLEY, M. P. The force that shape organizational performance measurement systems: an interdisciplinary review. International Journal of Production Economics, Amsterdam, v. 60, n. 61, p. 53-60, 1999.

WHITE, G. P. A survey and taxonomy of strategy-related performance measures for manufacturing. International Journal of Operations \& Production Management, Bradford, v. 16, n. 3, p. 24-61, 1996.

WONGRASSAMEE, S.; GARDINER, P. D.; SIMMONS, J. E. L. Performance measurement tools - the Balanced Scorecard and the EFQM excellence model. Measuring Business Excellence, Bradford, v. 7, n. 1, p. 14-29, 2003.

YIN, R. Case study research: design and methods. 2. ed. London: Sage, 1994. 170p.

\section{Sobre os autores}

\section{Gerusa Tinasi de Oliveira \\ Roberto Antonio Martins}

Departamento de Engenharia de Produção - DEP, Universidade Federal de São Carlos - UFSCar,

Rod. Washington Luís, Km 235, CEP 13565-905, São Carlos, SP, Brasil,

e-mails: gerusatinasi@yahoo.com.br; ram@dep.ufscar.br

Agradecimentos: Agradecemos ao Conselho Nacional de Desenvolvimento Científico e Tecnológico (CNPq) pelo apoio financeiro para desenvolvimento da pesquisa e à Coordenação de Aperfeiçoamento de Pessoal de Nível Superior (CAPES) pela bolsa de estudos concedida. 